\title{
The causal relationship between circulating cytokines and critically ill COVID-19: A bidirectional Mendelian randomization analysis
}

\author{
Yu Yang, Yang Xiaohui, Sui Miao, \\ Zhong Yingshuo \\ Endocrinology Department, Affiliated \\ Zhongshan Hospital of Dalian University, \\ Dalian, Liaoning, China
}

\begin{abstract}
Background In this study, we performed a bidirectional mendelian randomization analysis on circulating cytokines and critically ill COVID-19.

Methods Both the exposure and outcome data were obtained from public genome wide association study (GWAS) database. We extracted independent instrumental variables from exposure at genome level significance $\left(P<5 \times 10^{-8}\right)$. Wald ratio or inverse variance weighted (IVW) method were used for estimating the causal relationships between circulating cytokines and critically ill COVID-19.

Results Only IL5 (cytokines to critically ill COVID-19 direction) and bNGF, IL8 (critically ill COVID-19 to cytokines direction) showed suggestive causal relations. However, these associations lost significance after FDR correction. Another validation data set of critically ill COVID-19 did not confirm these associations, either.
\end{abstract}

Conclusions: Our Mendelian randomization did not find causal relationships between analyzable circulating cytokines and critically ill COVID-19.

The newly emerging COVID-19 (Corona virus disease 2019) is continuing to threaten the public health system throughout the world. The clinical manifestation of COVID-19 is complex, and substantial variations have been observed in the severity of disease. Most infected persons have mild or even no symptoms. However, the critically ill COVID-19 patients with severe respiratory failure and acute respiratory distress syndrome (ARDS) might need intensive therapy and have higher mortality rates [1].

Up to now, the exact pathogenesis of critically ill COVID-19 and its associated respiratory failure is poorly understood. Those individuals who are susceptible to severe infection and the immune mediated disease are strongly heritable and associated with specific genetic variants [2,3]. Several genome-wide association studies (GWAS) in critically ill COVID-19 demonstrated that, compared to the mild and moderate subtype, the severe COVID-19 might have a distinct pathophysiology [4,5]. COVID-19 infection is usually accompanied with an adverse host event "cytokine storm", which is characterized by an aggressive inflammatory response with the release of a large amount of pro-inflammatory cytokines [6-8]. Several studies have suggested that, the cytokine storm correlated directly with lung injury, multi-organ failure, and unfavorable prognosis of critically ill COVID-19 [8]. Moreover, effective strategies targeting cytokines during the treatment of severe COVID-19 patients could improve the survival rates and reduce mortality $[9,10]$. 
However, whether these cytokines have causal relationship with critically ill COVID-19 remains to be elucidated. The mendelian randomization (MR) approach, which uses genetic variants as instrumental variables, has been adopted as a powerful method to evaluate the causal relationship of potential exposure on certain disease [11]. The genetic variants, usually SNPs, are randomly assigned during gamete formation and less likely to be affected by confounding. Therefore, this kind of MR study is analogous to a randomized controlled trial, and can attain stable and convincing causal effects between exposures and outcomes [12,13].

A better understanding of the role of inflammatory biomarkers in COVID-19 is needed, which may help prevent the incidence and aid in the development of novel therapeutic targets. In this study, we performed a bidirectional MR analysis to assess the causal direction between cytokines with critically ill COVID-19 using GWAS summary data. The aim of the present study was to (1) estimate the causal relationships between cytokines and the development of critically ill COVID-19; (2) appraise the evidence for direction and robustness in the estimated etiological associations; (3) investigate potential genetic variants that lead to the variation of COVID-19 related cytokines, which might also contribute to the progress of critically ill COVID-19.

\section{METHODS}

\section{Study design}

In this study, we conducted a bidirectional MR test to decipher the causal effect of circulating cytokines with critically ill COVID-19 using publicly available genetic association summary data (two-sample MR). The underlying idea of the MR method is to use genetic variants as instrumental variables (IVs) to link a risk factor ('exposure') to a health trait ('outcome'). In order to represent a valid IV, a genetic variant need to fulfill three assumptions: 1) is associated with the exposure, 2) only affect an outcome via the exposure, 3) and is independent of confounders. In this MR study, exposures and outcomes in the cytokines to critically ill COVID-19 direction are circulating cytokines levels and COVID-19, respectively. For the critically ill COVID-19 to cytokines direction, the assignment is reversed.

Contributing studies have received ethical approval from their respective institutional review boards. This MR study is a secondary research from public available data. Therefore, no extra ethical approval and informed consent are required.

\section{Genetically determined levels of circulating cytokines}

The circulating cytokines summary data were obtained from Ahola-Olli's study [14]. In this study, the authors identified 27 genome-widely significant loci for 41 cytokines. For each cytokine, SNPs associated with circulating levels at a significance threshold $\left(P<5 \times 10^{-8}\right)$ were chosen as IVs. We performed linkage disequilibrium clumping for the significant SNPs through the European 1000 Genomes Project reference panel, and chose the independent genetic variants for further MR analysis. Overall, 24 cytokines with at least one genetic variable were identified as exposures for this study. Full GWAS summary statistics for the circulating cytokines are publicly available through the GWAS Catalog server at https://www.ebi.ac.uk/gwas/home.

\section{Covid-19 host genetics initiative (COVID-19 HG)}

The critically ill COVID-19 summary data were obtained from COVID-19 HG GWAS meta-analysis (https:// www.COVID-19hg.org/) [15]. In brief, the COVID-19 HG is an ongoing, international initiative that aims to facilitate COVID-19 host genetic study. After collecting pre-specified individual level or summary results from GWASs, it combines these data in an inverse variance weighted meta-analysis using variants with a minor allele frequency $(\mathrm{MAF})>0.001$ and imputation quality $\left(\mathrm{r}^{2}\right)>0.6$. In this study, we obtained the summary statistics from data R5A2 (European population), which collects critically ill COVID-19 $(\mathrm{n}=4792)$ and population controls $(n=1054664)$. For privacy issues, the summary data used in this study did not include the 23andme and UKBB cohorts.

\section{Statistical analysis}

The MR analysis and sensitivity analysis were conducted using R platform via R package TwoSampleMR [16]. We used the standard Wald ratio estimator (when only 1 IV exists) or inverse variance weighted (IVW) method (when several IVs are available) as the primary MR analysis for estimating the causal relationships between circulating cytokines and critically ill COVID-19 [17]. FDR (false discovery rate) correction was 
applied to correct for multiple comparisons, and the $P$ values after FDR correcttion were described as $P$. adjust. A $P<0.05$ before FDR correction was considered as suggestive for association.

The statistical power calculations for the MR analysis were performed through online tool at https://sb452. shinyapps.io/power/ [18]. Briefly, for the cytokines to critically ill COVID-19 direction, the outcome was binary. We computed the power to detect 1.2 causal effects per SD change in exposure for each MR analysis at a 0.05 significance level. While for the other direction, the outcomes were continuous. Causal effect per SD change of 0.2 at a 0.05 significance level was adopted for power estimation.

In order to assess the robustness of the MR results and potential violations of the assumptions underlying MR, we performed several kinds of sensitivity analyses. These sensitivity analyses mainly include heterogeneity tests, horizontal pleiotropy and leave-one-out analysis.

Heterogeneity test, which assess the compatibility of IVs in causal effects, is an important indicator of potential violation of IV assumptions [19]. We conducted Cochran's Q test and $I^{2}$ statistic to compute the heterogeneity of causal estimates from each genetic variant. The MR-Egger regression was performed to assess potential directional pleiotropy of genetic variants. The slope of the MR-Egger regression can give pleiotropy corrected causal estimates, and the magnitude of the intercept can provide an estimate of the degree of pleiotropy. If the intercept differs from zero, there is evidence of directional pleiotropy. In addition, the MR-PRESSO method was applied to detect and correct for the horizontal pleiotropic outlier. Briefly, it regresses the IV-outcome and IV-exposure association to conduct a global test of heterogeneity. Then, it compares the observed distance of each SNP from the regression with the distance expected under the null hypothesis of no pleiotropy. Finally, the leave-one-out analysis is performed to evaluate whether the MR estimate is biased by any single SNP that might have a substantial horizontal pleiotropic effect. In this method, we can re-estimate the effect by sequentially dropping one SNP at a time. If the dropped SNP lead to a dramatic change in the estimate, it may have horizontal pleiotropic effect.

\section{RESULTS}

\section{Instrument variables in this study}

In order to eliminate potential linkage disequilibrium (LD) of genetic variants, we performed strict clumping with an $\mathrm{R}^{2}$ threshold of 0.01 after extracting genome-wide associated SNPs $\left(P<5 \times 10^{-8}\right)$ from exposure GWAS data. Because the exposure and outcome population are both from European population, European samples in the 1000 genomes project reference data set is chosen as the reference panel for clumping.

In the cytokines to critically ill COVID-19 direction, 80 independent SNPs remained and were utilized as instruments for 24 cytokines following clumping (Table 1). Other 17 cytokines were not suitable for exposure due to lack of appropriate IVs. Among the 24 cytokines for exposure, 8 cytokines have only 1 associated SNP. While the other 16 cytokines were associated with multiple IVs, and MIPlb having the most 18 IVs. For the critically ill COVID-19 to cytokines direction, all the 41 cytokines were eligible for outcomes. After pruning strict linkage disequilibrium clumping and data harmonization, 8 independent variants remained for further MR analysis (Table 2, Table S1 in the Online Supplementary Document). Then, we calculated F statistics for all the instrument SNPs in our analysis to avoid the weak instrument bias [20]. As shown in Table 1 and Table 2, the F-statistics were well above the usual threshold of 10.

\section{Bidirectional Mendelian randomization analysis}

The primary results of our MR analysis for the circulating cytokines and critically ill COVID-19 were presented in Table 1 and Table 2. For the cytokines to critically ill COVID-19 direction, the only cytokine reaching statistically significant was IL5 $(P=0.030, \mathrm{OR}=0.674 ; 95 \%$ confidence interval $(\mathrm{CI})=0.472$ 0.963). However, this $P$ value lost significance following FDR correction for testing multiple cytokines (Padjust $=0.720$ ). All the other 23 cytokines did not show confirmative association with critically ill COVID-19.

For the critically ill COVID-19 to cytokines direction, we conducted 41 times MR analysis. IVW method was applied in this direction, for eight independent IVs are available. The bNGF and IL8 cytokines showed suggestive association, with $P$ value $0.044(\mathrm{OR}=0.911,95 \% \mathrm{CI}=0.832-0.998)$ and $0.049(\mathrm{OR}=0.913,95 \%$ $\mathrm{CI}=0.525-0.999)$, respectively. However, FDR correction for these $P$ values did not show significance ( $P$. adjust $=0.574$. 
Table 1. MR analysis results of 24 cytokines to critically ill COVID-19 direction*

\begin{tabular}{|c|c|c|c|c|c|c|c|}
\hline CYTOKINES & NUMBER OF SNPS & VARIANCE EXPLAINED & F STATISTICS & BETA & SE & P Value & PADJust \\
\hline CTACK & 2 & 0.049 & 184.615 & 0.055 & 0.086 & 0.526 & 0.789 \\
\hline bNGF & 1 & 0.011 & 39.017 & 0.016 & 0.229 & 0.946 & 0.946 \\
\hline VEGF & 7 & 0.045 & 320.340 & -0.094 & 0.057 & 0.103 & 0.786 \\
\hline TRAIL & 12 & 0.245 & 2074.603 & -0.071 & 0.044 & 0.106 & 0.786 \\
\hline $\mathrm{TNFb}$ & 2 & 0.104 & 173.738 & 0.066 & 0.090 & 0.462 & 0.786 \\
\hline SCGFb & 3 & 0.071 & 266.890 & 0.095 & 0.091 & 0.296 & 0.786 \\
\hline SCF & 2 & 0.010 & 87.294 & -0.443 & 0.311 & 0.155 & 0.786 \\
\hline IL16 & 3 & 0.092 & 342.450 & -0.005 & 0.063 & 0.936 & 0.946 \\
\hline RANTES & 1 & 0.004 & 13.974 & 0.249 & 0.342 & 0.467 & 0.786 \\
\hline PDGFbb & 6 & 0.070 & 592.388 & -0.080 & 0.076 & 0.291 & 0.786 \\
\hline MIPlb & 18 & 0.305 & 2630.814 & 0.062 & 0.066 & 0.344 & 0.786 \\
\hline MIG & 1 & 0.010 & 38.195 & -0.016 & 0.236 & 0.945 & 0.946 \\
\hline MCSF & 1 & 0.016 & 13.724 & -0.220 & 0.200 & 0.271 & 0.786 \\
\hline $\mathrm{MCPl}$ & 2 & 0.024 & 203.860 & -0.167 & 0.216 & 0.440 & 0.786 \\
\hline IL12p70 & 4 & 0.018 & 149.053 & 0.097 & 0.195 & 0.617 & 0.824 \\
\hline IP10 & 2 & 0.019 & 71.290 & -0.286 & 0.234 & 0.221 & 0.786 \\
\hline IL18 & 3 & 0.041 & 150.175 & 0.015 & 0.125 & 0.905 & 0.946 \\
\hline IL17 & 1 & 0.005 & 41.923 & -0.152 & 0.337 & 0.652 & 0.824 \\
\hline IL13 & 1 & 0.089 & 347.870 & -0.099 & 0.089 & 0.265 & 0.786 \\
\hline IL10 & 1 & 0.005 & 37.121 & -0.300 & 0.345 & 0.384 & 0.786 \\
\hline HGF & 2 & 0.015 & 128.368 & 0.067 & 0.143 & 0.636 & 0.824 \\
\hline IL5† & 1 & 0.011 & 38.656 & -0.395 & 0.182 & 0.030 & 0.720 \\
\hline GROa & 2 & 0.119 & 444.370 & -0.004 & 0.054 & 0.934 & 0.946 \\
\hline Eotaxin & 2 & 0.027 & 222.288 & -0.066 & 0.096 & 0.491 & 0.786 \\
\hline
\end{tabular}

$\mathrm{SE}-$ standard error, Padjust - FDR (false discovery rate) corrected P value

*Explained variance was calculated as: $\mathrm{R}^{2}=(\text { reported GWAS effect })^{2} \times 2 \times$ minor allele frequency $\times(1-$ minor allele frequency $) /$ variance of trait. F statistics per variant was calculated as: (Sample size -2$) \times R^{2} /\left(1-R^{2}\right)$. The Wald ratio estimator was used for effect estimation if one single instrument was available, and the inverse variance weighted estimation method (IVW) when more than one instrument was available.

$† P$ value of IL5 showed suggestive significance at the level of 0.05 .

Table 2. MR analysis results of critically ill COVID-19 to 41 cytokines direction

\begin{tabular}{|c|c|c|c|c|c|}
\hline CYTOKINES & NUMBER OF SNPS* & BETA & SE & P VALUE & PADJUST \\
\hline CTACK & 8 & 0.042 & 0.062 & 0.494 & 0.856 \\
\hline $\mathrm{bNGF} \dagger$ & 7 & -0.093 & 0.046 & 0.044 & 0.574 \\
\hline VEGF & 7 & -0.048 & 0.033 & 0.145 & 0.688 \\
\hline MIF & 7 & -0.023 & 0.047 & 0.628 & 0.888 \\
\hline TRAIL & 8 & -0.010 & 0.034 & 0.766 & 0.894 \\
\hline $\mathrm{TNFb}$ & 7 & -0.047 & 0.087 & 0.590 & 0.888 \\
\hline $\mathrm{TNFa}$ & 8 & -0.018 & 0.057 & 0.755 & 0.894 \\
\hline SDFla & 8 & -0.008 & 0.031 & 0.788 & 0.894 \\
\hline SCGFb & 7 & -0.060 & 0.059 & 0.306 & 0.856 \\
\hline SCF & 7 & -0.036 & 0.030 & 0.240 & 0.856 \\
\hline IL16 & 8 & 0.014 & 0.045 & 0.766 & 0.894 \\
\hline RANTES & 8 & 0.006 & 0.072 & 0.939 & 0.939 \\
\hline PDGFbb & 7 & 0.015 & 0.030 & 0.628 & 0.888 \\
\hline MIPlb & 8 & -0.026 & 0.041 & 0.520 & 0.856 \\
\hline MIPla & 7 & -0.040 & 0.046 & 0.390 & 0.856 \\
\hline MIG & 7 & -0.017 & 0.059 & 0.777 & 0.894 \\
\hline MCSF & 7 & -0.087 & 0.055 & 0.116 & 0.688 \\
\hline MCP3 & 8 & -0.126 & 0.088 & 0.151 & 0.688 \\
\hline $\mathrm{MCPl}$ & 7 & -0.033 & 0.030 & 0.278 & 0.856 \\
\hline IL12p70 & 6 & -0.029 & 0.034 & 0.393 & 0.856 \\
\hline IP10 & 6 & 0.038 & 0.069 & 0.575 & 0.888 \\
\hline IL18 & 7 & 0.015 & 0.046 & 0.749 & 0.894 \\
\hline IL17 & 8 & -0.037 & 0.031 & 0.225 & 0.856 \\
\hline IL13 & 7 & -0.047 & 0.046 & 0.311 & 0.856 \\
\hline IL10 & 7 & -0.060 & 0.031 & 0.056 & 0.574 \\
\hline IL8末 & 7 & -0.091 & 0.046 & 0.049 & 0.574 \\
\hline IL6 & 7 & -0.007 & 0.031 & 0.807 & 0.894 \\
\hline ILlra & 7 & -0.016 & 0.046 & 0.730 & 0.894 \\
\hline
\end{tabular}


Table 2. Continued

\begin{tabular}{cccccc} 
CYTOKINES & NUMBER OF SNPS* & BETA & SE & P VALUE & PADJUST \\
ILlb & 7 & -0.058 & 0.036 & 0.113 & 0.688 \\
\hline HGF & 7 & -0.058 & 0.030 & 0.055 & 0.574 \\
\hline IL9 & 8 & -0.004 & 0.045 & 0.920 & 0.939 \\
\hline IL7 & 7 & -0.036 & 0.047 & 0.445 & 0.856 \\
\hline IL5 & 7 & -0.073 & 0.047 & 0.121 & 0.688 \\
\hline IL4 & 7 & -0.026 & 0.031 & 0.395 & 0.856 \\
\hline IL2ra & 7 & -0.036 & 0.057 & 0.522 & 0.856 \\
\hline IL2 & 7 & -0.039 & 0.047 & 0.406 & 0.856 \\
\hline IFNg & 7 & 0.004 & 0.032 & 0.894 & 0.939 \\
\hline GROa & 7 & -0.075 & 0.078 & 0.334 & 0.856 \\
\hline GCSF & 8 & -0.003 & 0.030 & 0.910 & 0.939 \\
\hline FGFbasic & 8 & -0.023 & 0.031 & 0.452 & 0.856 \\
\hline Eotaxin & 7 & -0.020 & 0.031 & 0.522 & 0.856 \\
\hline
\end{tabular}

SNP - single nucleotide polymorphism, SE - standard error, Padjust - FDR (false discovery rate) corrected $P$ value

*The inverse variance weighted estimation method (IVW) was used in this direction, for six to eight SNPs were available.

$\dagger P$ value of bNGF showed suggestive significance at the level of 0.05 .

$¥ P$ value of IL8 showed suggestive significance at the level of 0.05 .

\section{Statistical power, heterogeneity and sensitivity tests}

The statistical power of our MR analysis was shown in the Table S2 in the Online Supplementary Document. In the cytokines to critically ill COVID-19 direction, two independent COVID-19 data sets with similar sample size and case proportion were enrolled in our study. For each COVID-19 data set, nearly half cytokines reached the power of $80 \%$. The other cytokines with lower power values were caused by lower explained phenotype variance. The two enrolled data sets would raise the power values in certain degree. However, we could not compute the exact power for the two data sets together, due to lack of raw GWAS data. For the suggestive cytokine IL5, the estimated power was $26.1 \%$ and $26.8 \%$, respectively. And the actual OR value were 0.674 and 0.646 in each data set, Therefore, the power to detect IL 5 association with critically ill COVID-19 would reach $81.5 \%$ and 89.5\%. In the critically ill COVID-19 to cytokines direction, power values were calculated based on the 8 independent SNPs (total explained variance 0.127) and sample size of 8293 [14]. At the causal effect per SD change in exposure of 0.2 and significance level of 0.05 , the power would reach $100 \%$. We performed heterogeneity and sensitivity tests for above suggestive association MR results. For the cytokines to critically ill COVID-19 direction, only IL5 showed suggestive association with COVID-19. Because there is only one IV (rs7767396) available for IL5, no heterogeneity and horizontal pleiotropy exist. As shown in the Table 1, this IV explained $1.2 \%$ of the variance of IL5 levels. Therefore, we queried rs 7767396 through phenoscanner database [21], which is a curated database holding publicly available results from large-scale genome-wide association studies. It is indicated that, rs7767396 is also associated with serum VEGF levels.

For the critically ill COVID-19 to cytokines direction, there are eight independent IVs in effect estimates. We conducted heterogeneity and sensitivity tests to exclude potential violation of MR assumptions (Table 3). Cochran's $Q$ indicated that, there are no evidence of heterogeneity for bNGF $(P=0.860)$ and IL8 $(P=0.983)$. Moreover, MR Egger regression revealed no evidence of directional pleiotropy for critically ill COVID-19 to bNGF (intercept $=-0.002, P=0.962)$ and IL8 ( intercept $=-0.005, P=0.904)$. The "leave-one-out" analysis showed the IVW causal association estimate was not driven by a single SNP, either (Figure S1 in the Online Supplementary Document).

\section{Secondary validation study}

We further performed a secondary validation study for bidirectional MR analysis based on another independent critically ill COVID-19 GWAS data set (R4A2) from COVID-19 HG (Tables S3, S4 in the Online Supplementary Document). This validation data set R4A2 consists of 4933 critically ill COVID-19 patients and 1398672 healthy controls. Table 4 showed the results of validation study for the suggestive causal association cytokines (IL5 in cytokines to COVID-19 direction, and bNGF, IL8 in COVID-19 to cytokines direction) discovered in R5A2 data set. In the cytokines to COVID-19 direction, IL5 also showed marginal association ( $P=0.019$, OR 0.646, 95\% CI 0.448-0.932). However, this association lost significance after FDR correction (P.adjust $=0.456$ ). The other two cytokines (bNGF, IL8) were not associated with critically ill COVID-19. The secondary validation study did not show confirmative association for these cytokines with critically ill COVID-19 in both directions. 
Table 3. Heterogeneity and pleiotropy tests for bNGF and IL8 IVs in the critically ill COVID-19 to cytokines direction

\begin{tabular}{lccccccc}
\multirow{2}{*}{ Outcomes } & \multicolumn{1}{c}{ HeterogeneItY TEST } & \multicolumn{3}{c}{ Pleiotropy teSt } \\
\multirow{2}{*}{ bNGF } & Method & Q value & $\mathbf{I}^{2}$ & $\boldsymbol{P}$ value & Egger intercept & SE & $P$ value \\
\cline { 2 - 8 } & MR-Egger & 2.576 & 1.000 & 0.765 & -0.002 & 0.043 & 0.962 \\
\hline \multirow{2}{*}{ IL8 } & IVW & 2.578 & 1.388 & 0.860 & & & 0.904 \\
\hline
\end{tabular}

SE - standard error

Table 4. Validation study of MR results in another COVID-19 data set

\begin{tabular}{lccccccc} 
EXPOSURES & OUtCOMES & BEtA & SE & P VALUE & OR & $95 \%$ Cl & PADJUST \\
IL5 & Critically ill COVID-19 & -0.437 & 0.187 & $0.019^{*}$ & 0.646 & $0.448,0.932$ & 0.456 \\
\hline Critically ill COVID-19 & bNGF & -0.088 & 0.050 & 0.080 & & & \\
\hline Critically ill COVID-19 & IL8 & -0.087 & 0.050 & 0.081 & & & \\
\hline
\end{tabular}

MR - Mendelian randomization, OR - odds ratio, $\mathrm{CI}$ - confidence interval

*P value of IL5 showed suggestive significance at the level of 0.05 in the cytokine to critically ill COVID-19 direction.

\section{DISCUSSION}

To our knowledge, this is the first attempt to assess the causal effect of the circulating cytokines levels and critically ill COVID-19 through two-sample bidirectional MR approach. Our results provide evidence that there is no confirmative causal effect between analyzable cytokines and critically ill COVID-19 in bilateral directions.

While the majority affected individuals have mild or even no symptoms, severe COVID-19 is more likely in the elderly population and those with comorbidities [1]. Cumulative studies show that, the severe COVID-19 is qualitatively different from mild or moderate disease. The exact pathogenesis underlying the critically ill COVID-19 patients remain unclear. Host genetic variations may explain part of the heterogeneity for different COVID-19 outcomes. At least two biological components, susceptibility to viral infection and propensity to develop harmful pulmonary inflammation, participate in the pathogenesis of COVID-19 mortality risk [3].

Therefore, interpreting the genetic role for critically ill COVID-19 may provide pivotal insights into its pathogenesis. The published observational studies have addressed the pivotal role of aberrant cytokines affecting inflammatory injuries of the lungs, lung blood vessels or even the general organs during critically ill COVID-19 patients. However, confounding factors (smoking, lifestyle, age, obesity and T2DM), which might bias the observed estimates, are difficult to be excluded in these observational studies. The genetic IVs for MR studies are randomly allocated during gemmate formation, and less affected by confounding factors. Therefore, the MR study has become an established method for elucidating causal relationship between the exposures and outcomes in observational epidemiology.

In the present study, we identified suggestive causal associations for IL5, bNGF and IL8 with critically ill COVID-19. However, the results from the dual directions provided no causal relationship between serum cytokines and critically COVID-19. Cochran's Q test suggested no heterogeneity among the IVs, and MR Egger regression result did not show any bias of directional pleiotropy. Moreover, another data set also validated the causal results. Therefore, bias may not exist in our MR analysis, and none of the three main MR assumptions was violated. Compared with other common diseases, the critically ill COVID-19 GWAS had moderate sample size, which would produce moderate power in detecting causal effects. In this study, the power of critically ill COVID-19 to cytokines direction was $100 \%$. While in the other direction, the power of about half cytokines did not reach $80 \%$. We further performed a validation study through an independent data set, which would raise the power in certain degree.

Although we did not demonstrate the confirmative causal effect of cytokines with critically COVID-19, these results did not repudiate the role of cytokine storm during the pathogenesis of critically ill COVID-19. In fact, more and more observational studies demonstrated the aberrant cytokines profile among COVID-19 patients compared to normal control individuals [22-25]. The cytokine storm might be an important mediator during severe lung injury. One reason for these discrepancies might be that, cytokine storm is not the specific pathogenesis for critically ill COVID-19. As a kind of overwhelming systemic inflammatory response, cytokine storm also occurs in several pathologic conditions, such as viral infection, cancer, severe sepsis and multi-organ failure [26]. MR study is mainly designated to extrapolate the causal association between the exposures and outcomes. Therefore, cytokine storm might be the manifestation of aberrant inner environment during critically 
ill COVID-19, rather than the causal reason. Another possible mechanism for lack of causal association is that, these cytokines might form a scale-free network to participate in the pathogenesis of critically ill COVID-19. These cytokines might have minor effect alone, but exert major effects when taking together, which are similar to polygenic risk score in complex traits. This assumption requires to be validated in the future study. Moreover, earlier study has revealed that, most cytokines levels, except ILl, peaked after respiratory function nadir, indicating that cytokine expression might not be the primary cause of impaired respiratory function in patients with COVID-19 [27]. And the dynamic cytokine storms and T cell lymphopenia are associated with COVID-19 severity. Sampling and measuring cytokines at one time might not reflex the dynamic fluctuations during critically ill COVID-19.

Our results might have certain clinical and public indications. As mentioned above, cytokine storm might be a common pathway in several clinical traits. SARS-Cov-2 infection might trigger aberrant cytokine profile in susceptible individuals, especially elderly persons with multiple organ failure. Effective protection and therapy for SARS-Cov-2 infection in these susceptible persons would be vital to beneficial prognosis. Therefore, the clinical decision to COVID-19 patients should be based on their personal profile and severity. It has been reported that, an immunologic memory of cytokine storm would likely persist, even after the SARS-CoV-2 infection resolves [28]. This adverse memory would raise the possibility of viral relapse or re-infection. We recommend longitudinal follow-up of COVID-19 patients with and without cytokine storm to decipher the immunological mechanisms and biomarkers for critically ill type. Our study has several limitations. First, the critically ill COVID-19 GWAS data of this study was obtained from COVID-19 HG. Compared to other traits, the coivd-19 HG has moderate sample size in COVID-19 patients. This would produce moderate power to detect the causal effect. The cumulative GWAS for COVID-19 might provide more powerful and convincible results. Second, we could not acquire independent instrument variables for 17 cytokines, including three most important pro-inflammatory cytokines of innate immune response, IL1, TNF $\alpha$, and IL6. Thus, the causal association of these cytokines and COVID-19 could not be assessed. Third, the exposure and outcome population of this study are European. Other ethic data are not available yet.

\section{CONCLUSIONS}

In conclusion, in our bidirectional MR study, no causal effect was detected between analyzable circulating cytokines and COVID-19. And the association between critically ill COVID-19 and cytokine storm need further consideration.

Acknowledgements: The authors would like to acknowledge the Ahola-Olli group and the COVID-19 host genetics initiative for their GWAS data. Contributing studies have received ethical approval from their respective institutional review boards. This MR study is a secondary research from publicly available data. Therefore, no extra ethical approval and informed consent are required.

Funding: None.

Authorship contributions: YY and ZY designed the study and wrote the main manuscript text. YX and SM prepared figures and tables. All the authors reviewed the manuscript.

Competing interests: The authors completed the Unified Competing Interest Form (available upon request from the corresponding author), and declare no conflicts of interest.

\section{Additional material}

Online Supplementary Document

1 Gao YD, Ding M, Dong X, Zhang JJ, Kursat Azkur A, Azkur D, et al. Risk factors for severe and critically ill COVID-19 patients. Allergy. 2021;76:428-55. Medline:33185910 doi:10.1111/all.14657

2 Zeberg H, Paabo S. The major genetic risk factor for severe COVID-19 is inherited from Neanderthals. Nature. 2020;587:6102. Medline:32998156 doi:10.1038/s41586-020-2818-3

3 Ovsyannikova IG, Haralambieva IH, Crooke SN, Poland GA, Kennedy RB. The role of host genetics in the immune response to SARS-CoV-2 and COVID-19 susceptibility and severity. Immunol Rev. 2020;296:205-19. Medline:32658335 doi:10.1111/ imr. 12897

4 Severe Covid-19 GWAS Group; Ellinghaus D, Degenhardt F, Bujanda L, Buti M, Albillos A, et al. Genomewide Association Study of Severe Covid-19 with Respiratory Failure. N Engl J Med. 2020;383:1522-34. Medline:32558485 doi:10.1056/NEJMoa2020283 
5 Pairo-Castineira E, Clohisey S, Klaric L, Bretherick AD, Rawlik K, Pasko D, et al. Genetic mechanisms of critical illness in COVID-19. Nature. 2021;591:92-8. Medline:33307546 doi:10.1038/s41586-020-03065-y

6 McCoy K, Peterson A, Tian Y, Sang Y. Immunogenetic Association Underlying Severe COVID-19. Vaccines (Basel). 2020;8:700. Medline:33233531 doi:10.3390/vaccines8040700

7 Hu B, Huang S, Yin L. The cytokine storm and COVID-19. J Med Virol. 2021;93:250-6. Medline:32592501 doi:10.1002/ jmv. 26232

8 Ye Q, Wang B, Mao J. The pathogenesis and treatment of the 'Cytokine Storm' in COVID-19. J Infect. 2020;80:607-13. Medline:32283152 doi:10.1016/j.jinf.2020.03.037

9 Yessayan L, Szamosfalvi B, Napolitano L, Singer B, Kurabayashi K, Song Y, et al. Treatment of Cytokine Storm in COVID-19 Patients With Immunomodulatory Therapy. ASAIO J. 2020;66:1079-83. Medline:33136592 doi:10.1097/MAT.0000000000001239

10 Shadvar K, Tagizadiyeh A, Gamari AA, Soleimanpour H, Mahmoodpoor A. Hemoperfusion as a Potential Treatment for Critically Ill COVID-19 Patients with Cytokine Storm. Blood Purif. 2021;50:405-7. Medline:33171470 doi:10.1159/000511391

11 Lawlor DA, Harbord RM, Sterne JA, Timpson N, Davey Smith G. Mendelian randomization: using genes as instruments for making causal inferences in epidemiology. Stat Med. 2008;27:1133-63. Medline:17886233 doi:10.1002/sim.3034

12 Zoccali C. The challenge of Mendelian randomization approach. Curr Med Res Opin. 2017;33:5-8. Medline:28952387 doi:1 $0.1080 / 03007995.2017 .1378514$

13 Zheng J, Baird D, Borges MC, Bowden J, Hemani G, Haycock P, et al. Recent Developments in Mendelian Randomization Studies. Curr Epidemiol Rep. 2017;4:330-45. Medline:29226067 doi:10.1007/s40471-017-0128-6

14 Ahola-Olli AV, Wurtz P, Havulinna AS, Aalto K, Pitkanen N, Lehtimaki T, et al. Genome-wide Association Study Identifies 27 Loci Influencing Concentrations of Circulating Cytokines and Growth Factors. Am J Hum Genet. 2017;100:40-50. Medline:27989323 doi:10.1016/j.ajhg.2016.11.007

15 Initiative C-HG. The COVID-19 Host Genetics Initiative, a global initiative to elucidate the role of host genetic factors in susceptibility and severity of the SARS-CoV-2 virus pandemic. Eur J Hum Genet. 2020;28:715-8. Medline:32404885 doi:10.1038/ s41431-020-0636-6

16 Hemani G, Zheng J, Elsworth B, Wade KH, Haberland V, Baird D, et al. The MR-Base platform supports systematic causal inference across the human phenome. eLife. 2018;7:e34408. Medline:29846171 doi:10.7554/eLife.34408

17 Burgess S, Butterworth A, Thompson SG. Mendelian randomization analysis with multiple genetic variants using summarized data. Genet Epidemiol. 2013;37:658-65. Medline:24114802 doi:10.1002/gepi.21758

18 Burgess S. Sample size and power calculations in Mendelian randomization with a single instrumental variable and a binary outcome. Int J Epidemiol. 2014;43:922-9. Medline:24608958 doi:10.1093/ije/dyu005

19 Burgess S, Bowden J, Fall T, Ingelsson E, Thompson SG. Sensitivity Analyses for Robust Causal Inference from Mendelian Randomization Analyses with Multiple Genetic Variants. Epidemiology. 2017;28:30-42. Medline:27749700 doi:10.1097/ EDE.0000000000000559

20 Burgess S, Thompson SG, Collaboration CCG. Avoiding bias from weak instruments in Mendelian randomization studies. Int J Epidemiol. 2011;40:755-64. Medline:21414999 doi:10.1093/ije/dyr036

21 Kamat MA, Blackshaw JA, Young R, Surendran P, Burgess S, Danesh J, et al. PhenoScanner V2: an expanded tool for searching human genotype-phenotype associations. Bioinformatics. 2019;35:4851-3. Medline:31233103 doi:10.1093/bioinformatics/btz469

22 Wilk AJ, Rustagi A, Zhao NQ, Roque J, Martinez-Colon GJ, McKechnie JL, et al. A single-cell atlas of the peripheral immune response in patients with severe COVID-19. Nat Med. 2020;26:1070-6. Medline:32514174 doi:10.1038/s41591-020-0944-y

23 Han H, Ma Q, Li C, Liu R, Zhao L, Wang W, et al. Profiling serum cytokines in COVID-19 patients reveals IL-6 and IL-10 are disease severity predictors. Emerg Microbes Infect. 2020;9:1123-30. Medline:32475230 doi:10.1080/22221751.2020.1770129

24 Mathew D, Giles JR, Baxter AE, Oldridge DA, Greenplate AR, Wu JE, et al. Deep immune profiling of COVID-19 patients reveals distinct immunotypes with therapeutic implications. Science. 2020;369:eabc8511. Medline:32669297 doi:10.1126/science.abc8511

25 McElvaney OJ, McEvoy NL, McElvaney OF, Carroll TP, Murphy MP, Dunlea DM, et al. Characterization of the Inflammatory Response to Severe COVID-19 Illness. Am J Respir Crit Care Med. 2020;202:812-21. Medline:32584597 doi:10.1164/rccm.202005-15830C

26 de la Rica R, Borges M, Gonzalez-Freire M. COVID-19: In the Eye of the Cytokine Storm. Front Immunol. 2020;11:558898. Medline:33072097 doi:10.3389/fimmu.2020.558898

27 Ong EZ, Chan YFZ, Leong WY, Lee NMY, Kalimuddin S, Haja Mohideen SM, et al. A Dynamic Immune Response Shapes COVID-19 Progression. Cell Host Microbe. 2020;27:879-882.e2. Medline:32359396 doi:10.1016/j.chom.2020.03.021

28 Bhaskar S, Sinha A, Banach M, Mittoo S, Weissert R, Kass JS, et al. Cytokine Storm in COVID-19-Immunopathological Mechanisms, Clinical Considerations, and Therapeutic Approaches: The REPROGRAM Consortium Position Paper. Front Immunol. 2020;11:1648. Medline:32754159 doi:10.3389/fimmu.2020.01648 